\title{
Etre malade sans diagnostic, difficile...
}

\author{
Jean Martin \\ Dr med., membre de la rédaction
}

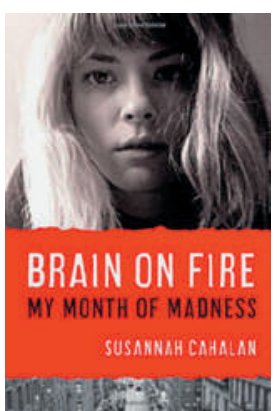

Susannah Cahalan Brain on Fire

My Month of Madness New York: Simon and Schuster; 2013. 273 pages. 17. 99 EUR. ISBN 978-1-451-62137-2
Susannah Cahalan est une journaliste du New York Post qui au début 2009, à 24 ans, a vécu une période de troubles d'allure neuro-psychiatrique qui ont été une énigme pour le New York University Langone Medical Center et sa division d'épileptologie. Après de multiples examens infructueux, c'est une biopsie de cerveau qui a permis aux médecins (notamment les neuropathologistes S. Najjar et J. Dalmau) de conclure à une maladie auto-immune, la Anti-NMDA receptor encephalitis, susceptible d'amélioration par une thérapeutique comprenant des stéroïdes, une plasmaphérèse et des immunoglobulines par voie intraveineuse (plus l'ablation d'un tératome le cas échéant - une moitié des cas en sont porteurs). Cahalan dit avoir été le $217^{\mathrm{e}}$ patient décrit, depuis lors des milliers l'ont été et plusieurs fondations consacrées à ce type de maladies ont vu le jour*; le «First International Symposium on Autoimmune Encephalitis» s'est tenu en mars 2014 à Durham, North Carolina - avec pour but de développer un consensus sur une définition et des critères diagnostiques.

Après quelques semaines de comportements surprenants et un "mois de folie», et une fois le traitement instauré, Susannah C. s'est progressivement rétablie et a repris son métier, quelque huit mois plus tard. Elle a voulu écrire son histoire et a fait un travail fouillé de recherche: parmi ses proches, en fonction de leurs témoignages et de notes qu'ils avaient rédigées alors (elle-même n'a pas de souvenir de la période critique), en interrogeant les médecins et étudiant son dossier, en s'intéressant à la littérature scientifique. La plus grande partie du texte est la description, parfois anecdotique, de sa vie depuis des premières manifestations bizarres, puis des crises épileptiformes ou de type psychotique. L'aggravation de son état demande l'hospitalisation. Une biopsie de cerveau donne la réponse et un traitement d'immuno-modulation est mis en œuvre. Ce livre et des interviews très médiatisés ont eu d'importants retentissements et ont fait mieux connaître les encéphalites auto-immunes. Des centaines de personnes/familles se sont adressées à l'auteure en racontant leurs parcours, marqués eux aussi par les incertitudes médicales, les transferts d'un service à l'autre (neurologie, psychiatrie), le manque de connaissances sur ces maladies et leur traitement. Ces contacts ont parfois eu des aspects dramatiques, quand par exemple le malade à propos de qui on l'appelait décède peu après.

La dernière partie de l'ouvrage aborde des problématiques plus générales, dans plusieurs chapitres dont un est intitulé «Survivor's Guilt». A juste titre sans doute mais des évaluations quantitatives seraient bien difficiles, Cahalan se demande combien de (milliers de) patients dans le passé on été étiquetés de schizophrénie ou de maladies dégénératives diverses alors qu'elles souffraient d'une telle encéphalite. Elle discute les situations lourdes, frustrantes, de personnes présentant une maladie manifestement grave mais où on ne parvient pas à poser de diagnostic (le livre est dédicacé aux patients sans diagnostic). Suite aux échos médiatiques, elle relève le soulagement exprimé par les personnes et milieux concernés: de ce que le sujet bénéficie d'attention et de ce que l'information à son égard devienne disponible en plus grande qualité et quantité.

Deux citations: "Je vis avec ce refrain constant: pourquoi mon corps a-t-il décidé de se retourner contre luimême? Pourquoi chez ceux qui sont touchés et pas les autres?» Et, à propos du premier médecin consulté qui a jugé qu'elle était sous l'effet d'un sevrage aigu d'alcool: «Ce neurologue connu n'avait jamais entendu parler de cette maladie. En quelque sorte, il est l'exemple de ce qui ne va pas en médecine. Il est le produit d'un système qui oblige les médecins à ne passer que quelques minutes avec chaque patient. Je peux me considérer heureuse, alors que mon cas était exceptionnel et demandait patience et attention individualisée, de n'avoir pas été victime de cette situation. Je réalise que ma guérison et maintenant ma capacité d'écrire ce livre, c'est cela qui est étonnant». Il ne s'agit pas de jeter indûment le blâme mais le fait est que les porteurs d'affections méconnues ont probablement besoin, plus souvent que d'autres, d'une dose de chance pour que le diagnostic soit posé et que la bonne thérapeutique soit entreprise en temps utile. 